\title{
Thermally Induced Aggregation of Rigid Spheres on a Liquid Surface
}

\author{
Eric Forgoston ${ }^{1}$, Leo Hentschker ${ }^{2}$, Siobhan Soltau ${ }^{1}$, \\ Patrick Truitt ${ }^{1}$ and Ashwin Vaidya ${ }^{1 *}$ \\ ${ }^{1}$ Complex Fluids Laboratory, Department of Mathematical Sciences, \\ Montclair State University, Montclair, NJ 07043, USA. \\ Department of Physics, Harvard University, 17, \\ Oxford Street, Cambridge, MA 02138, USA
}

October 22, 2015

\begin{abstract}
Fluids provide the optimal setting to explore natural patterns far from thermodynamic equilibrium. Experiments suggest that randomly dispersed particles on a liquid surface tend to aggregate on the surface of liquid over time, and the process is enhanced by an increase in the temperature of the liquid. We show that the agglomeration radii increases monotonically with temperature up until the point where all particles in the system form a single, large aggregate. The aggregation dynamics is related to changes in the material properties of the liquid including its viscosity and surface tension as well as the convection driven flow generated on the fluid surface. In this article we compare our experimental observations with analytical asymptotic results.

The analytical arguments are seen to agree well with the experimental observations.
\end{abstract}

Keywords: Aggregation, Convection, Capillary, Temperature, Surface tension

\section{Introduction}

Fluid dynamicists are well aware that the kitchen can be a fertile testing ground for important scientific discoveries. One such significant fluid dynamics phenomenon reveals itself in the simple act of making traditional Indian chai on the stove. Randomly distributed tea leaves on the surface of water are seen to aggregate into an increasingly compact configuration as the temperature of the water is increased (see Fig. 1). The temperature, although not the cause of the aggregation, serves to influence the time scale over which aggregation occurs. Indeed,

${ }^{*}$ Corresponding Author, Phone: 973.655.2138, Fax: 973.655.7686, Email: vaidyaa@mail.montclair.edu. 
aggregation has been noted to happen in cases when the system is in (or close to) thermal equilibrium.

The "cheerio effect" whereby cereal in a bowl of milk aggregates in a similar manner to the tea leaves has regained some recent attention in the literature [1]. The earliest contribution to the subject of aggregation of floating particles can be attributed to the 1949 paper by Nicholson [2]. Since then the most pertinent analytical contributions to the subject are due to Gifford and Scriven [3], Chan, Henry and White [4] and more recently by Vella and Mahadevan [1]. Their analysis is based on asymptotic arguments for capillary forces between spheres and/or cylinders. Additionally, Singh and Joseph [5] and Dixit and Homsy [6] have conducted rigorous numerical simulations to capture the effect of aggregation between multiple floating bodies. All of these works however, disregard the effect of temperature.

Interfacial aggregation is fairly widespread and has been observed in a variety of other settings $[7,8,9,10,11,12]$. The solid state physics and biophysical communities have long been aware of the effects of temperature upon interfacial aggregation and are deeply interested in the regulation of this behavior. Self assembled monolayers (SAMs) involve the construction of highly ordered materials by utilizing the local forces between disordered molecules and are used in the design and production of semiconductor nano-electronic devices including nano-wires, nano-tubes and nano-sensors [13, 14]. Several recent studies on SAMs report that temperature can be utilized to effectively control the topology of the networks formed $[13,15,16,17,18,19]$, create more stable structures and prevent the formation of cracks by a suitable sintering pretreatment which involves compactification and exposure to heat prior to the self-assembly process [20]. Temperature is also seen to impact the optical properties of the SAMs by suitable modification of their refractive indices [20]. While there is a lot written about aggregation and its potential applications, the effect of temperature on the aggregation process has never been explained in the literature from a fundamental physics perspective. Such a discussion is therefore significant and timely.

The current paper is devoted to the experimental study of interfacial particle aggrega-

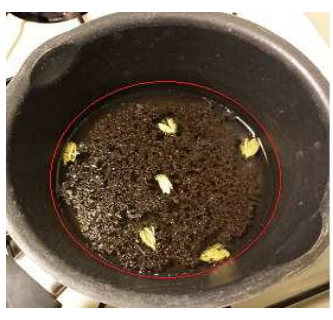

(a)

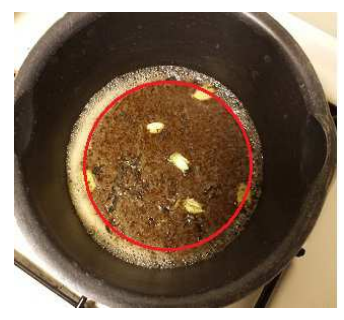

(b)

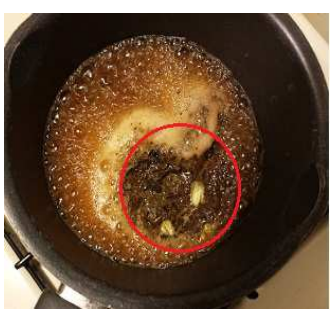

(c)

Figure 1: Aggregation of tea leaves on heated water. The tea leaves evolve from (a) randomly distributed to (b) somewhat aggregated to (c) tightly aggregated very quickly as the water temperature is raised. It is noted that this was not a controlled experiment and that our observations with tea leaves are somewhat different from the investigation in the paper, due to the absorption properties of the tea leaves, lack of temperature control and low viscosity of water in which tea is prepared. 


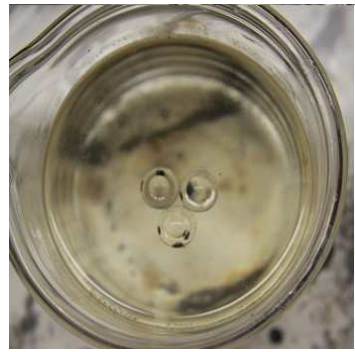

(a)

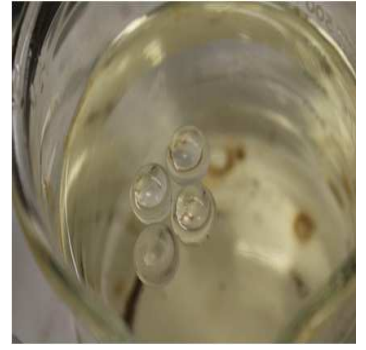

(b)

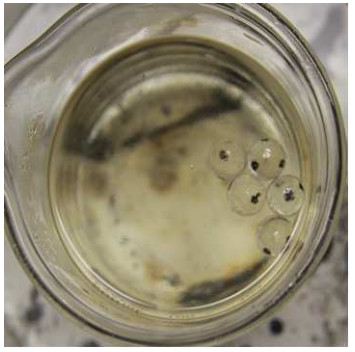

(c)

Figure 2: Aggregation of multiple spheres on a heated liquid surface. In (a) the water-corn syrup mixture was heated to $60^{\circ} \mathrm{C}$ while in (b) and (c) the mixture was heated to $70^{\circ} \mathrm{C}$.

tion under the influence of temperature. We hypothesize that the change in temperature of the fluid has a strong influence upon its material properties (viscosity and surface tension) thereby impacting the aggregation dynamics. Details of the experiments are explained in the proceeding sections. Our empirical models are used to extend the asymptotic expressions for aggregation time and the analytical results are shown to agree well with the experimental observations.

\section{Experiments}

\subsection{Methodology}

Our experiments were conducted in a beaker (diameter 4.0in, height 4.75in) filled with a solution containing equal volumes of water and corn syrup on a hot plate. In order to prevent heat loss, the beaker was immersed in a thermal bath. The beaker was then heated to a fixed temperature. After the desired temperature was reached, a number of acrylic spheres were placed into the solution and the time taken for the spheres to aggregate, henceforth denoted $t_{a}$, was recorded (see Fig. 2). Over the entire study the effect of temperature, sphere size and sphere quantity upon $t_{a}$ was investigated. In each experiment, the temperature was chosen to be within the range of $24^{\circ} \mathrm{C}-100^{\circ} \mathrm{C}$; two, three and four spheres of diameters $0.635 \mathrm{~cm}$, $1.27 \mathrm{~cm}$ and $2.54 \mathrm{~cm}$ were chosen for the study. The experiments were conducted with the spheres being placed at different initial positions in each of the experimental trials and each reported value of $t_{a}$ is the result of averaging three to five different trials.

The temperature distribution in the water-corn syrup sample was measured at various settings of the hot plate between $30^{\circ} \mathrm{C}$ and $100^{\circ} \mathrm{C}$. Specifically, after setting the hot plate to a fixed temperature and upon reaching equilibrium, thermometers were placed at three different radii (center of the beaker, $\frac{r}{3}$ and $\frac{2 r}{3}$, where $r$ is the radius of the beaker) and three different heights $(3.18 \mathrm{~cm}, 6.36 \mathrm{~cm}, 9.52 \mathrm{~cm})$ from the bottom. The observations revealed a very homogeneous distribution of temperature in the sample liquid with maximum standard deviation of $3^{\circ} \mathrm{C}$ from the mean temperature measurement from all the trials. For the most part, the deviations were considerably lower than this. 


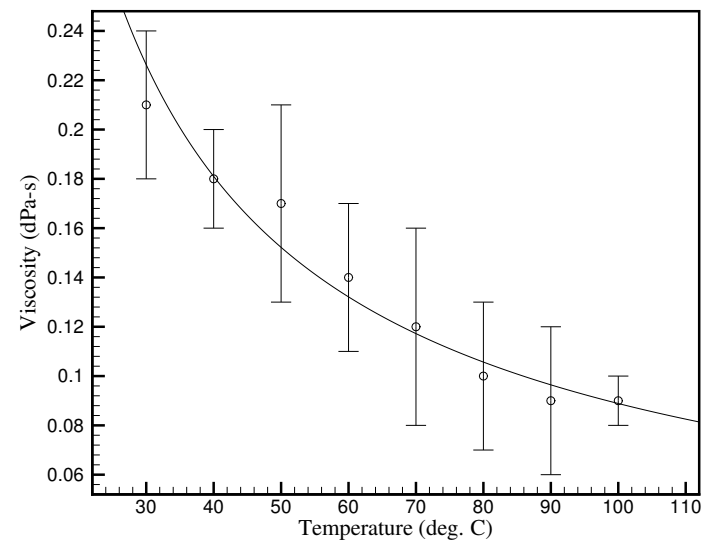

(a)

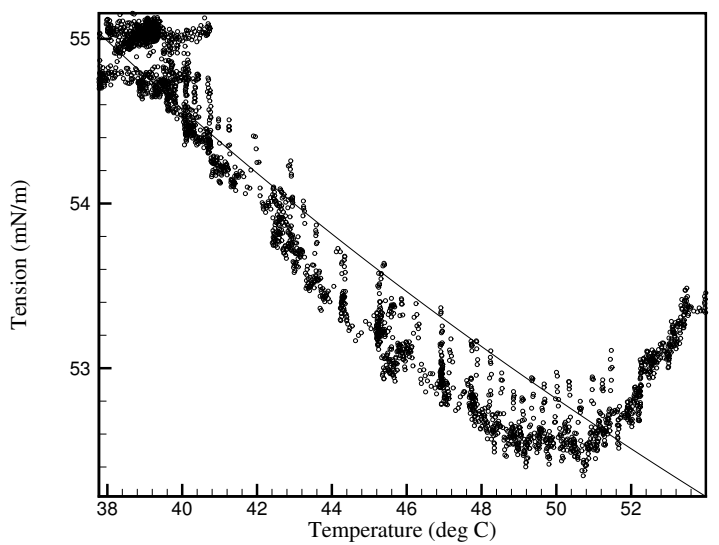

(b)

Figure 3: Measurements of (a) viscosity and (b) surface tension as a function of temperature $\theta$. The fitted curves to the experimental data show a declining trend in these material parameters with increasing $\theta$. The rising trend in (b) beyond $50^{\circ} \mathrm{C}$ is a result of experimental error as explained in the text.

The viscosity $\mu$ of the liquid was measured using a hand held rotational viscometer (Haake Rotational Viscometer). Viscosity is well known to be inversely proportional to the liquid's temperature [21]. Although we expected our sample solution to possess a similar profile, the exact nature of this dependence was ascertained. Here as well, the liquid was maintained at different fixed temperatures and several repetitions of the viscosity measurements were made. All experiments yielded a consistently decaying profile with a rise in temperature. A sample curve of viscosity versus temperature is shown in Fig. 3(a). A power law fit to the profile yields the relationship $\mu(\theta)=3.16 \theta^{-0.775}$, where $\theta$ is the temperature in ${ }^{\circ} \mathrm{C}$, with a correlation coefficient of $R^{2}=0.99$ demonstrating an excellent fit.

We also measured the surface tension $\gamma$ of the liquid as a function of temperature. For this purpose, a tensiometer (DeltaPi model, Kibron Inc.) was employed. The instrument was calibrated with distilled water and measurements were made by placing the beaker containing the sample liquid on a hot plate in a thermal bath. The hot plate was heated to approximately $70^{\circ} \mathrm{C}$ and the temperature and surface tension of the sample were simultaneously recorded (see Fig. 3(b)). Multiple measurements of the sample were made over a period of several months, and all measurements, for the most part, showed a declining trend in $\gamma$ as a function of temperature. The surface tension measurements were wrought with problems, including bubble formations, dust on the liquid surface, crystallization of corn syrup at higher temperature and possibly rapid evaporation, leading to errors in several attempts. Small variations between different samples of the liquid could also contribute to the observed variations. Precautions were taken to minimize the contributions of these factors. In particular, to minimize the negative and drastic impact of evaporation, it was deemed best to measure $\gamma$ in incremental ranges of temperature rather than considering the entire temperature range 


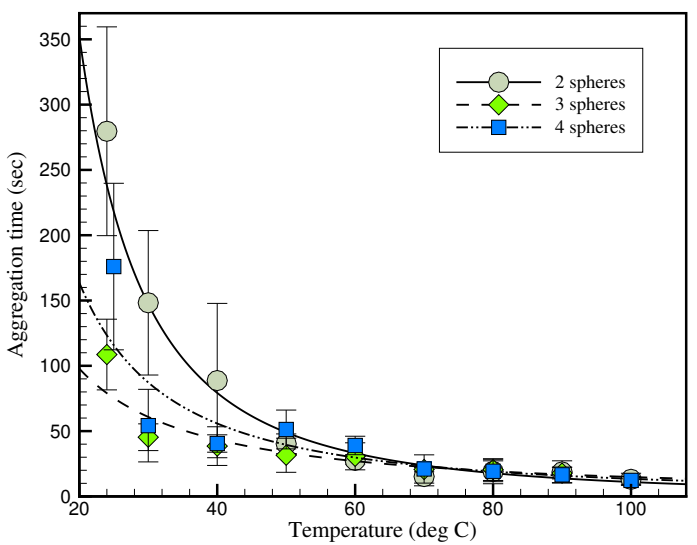

(a)

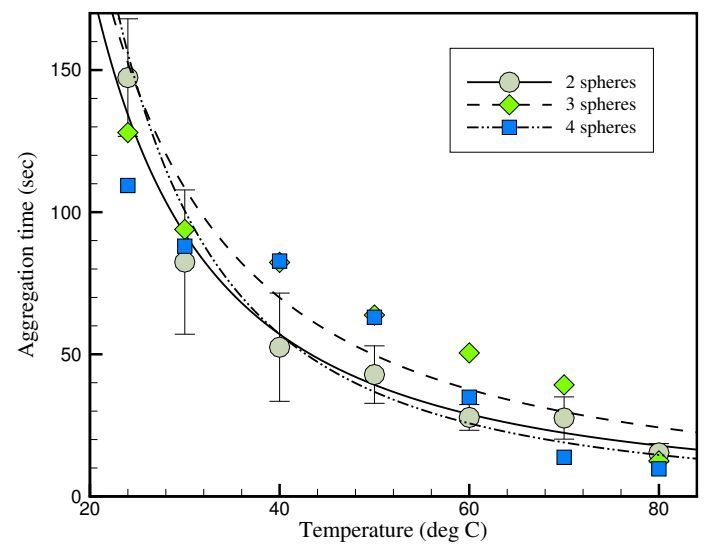

(b)

Figure 4: The plot shows the aggregation time as a function of temperature for (a) half-inch diameter spheres and (b) one-inch diameter spheres. The power law fits to the experimental data show strong $R^{2}$ correlations between 0.8-0.96.

explored in the aggregation study. It is however, unclear to us if evaporation by itself is a problem or a natural route through which temperature impacts surface surface tension. Our repeated experiments did confirm an inverse relationship between $\gamma$ and $\theta$ with power law fit exponents ranging between -0.0083 and -0.35 . In the sample shown in Fig. 3(b) the power law fit yields $\gamma(\theta)=107.4 \theta^{-0.183}$. Here, the rising trend beyond $50^{\circ} \mathrm{C}$ is known to be erroneous and such a trend was not observed during other runs for smaller ranges of temperatures. On an average, we find that surface tension declines at the rate of $0.44 \frac{N}{m \circ C}$. The overall qualitative trend is in keeping with surface tension versus temperature measurements for water and other liquids $[22,23]$. We are also aware of the other classical equations which model the surface tension-temperature relation [24] but have adopted the power-law fit in this study to allow for a simple comparison between experiments and asymptotic analysis.

\section{$2.2 \quad$ Results}

In our experiments we measured the characteristic time (or aggregation time) for the particles to form a single aggregate, i.e. when the particles first come into contact collectively. Figure 4 shows the characteristic time as a function of temperature. From this graph we can see that as the temperature of the solution increases the characteristic time decreases as aggregates form at a faster rate. We hypothesize that this decreased time is a result of the more powerful convection currents that occur at higher temperatures. This trend was seen to hold for differing numbers of spheres placed inside of the beaker. A power law fit, of the type $t_{a}=a \theta^{b}$ to the data obtained shows a good correlation but with minor variations depending upon the number and size of the spheres. The values of the fit parameter $b$ for the different cases are shown in Table 1 (with $R^{2}$ values ranging between $0.8-0.96$ ). 


\begin{tabular}{c|c|c|c}
\hline Diameter(in) & 2-spheres & 3-spheres & 4-spheres \\
\hline 0.5 & -2.15 & -1.17 & -1.55 \\
1.0 & -1.67 & -1.53 & -1.87 \\
\hline
\end{tabular}

Table 1: The table depicts the power law fit exponent $b$ corresponding to the $t=a \theta^{b}$ fits shown in Fig. 4(a)-(b).

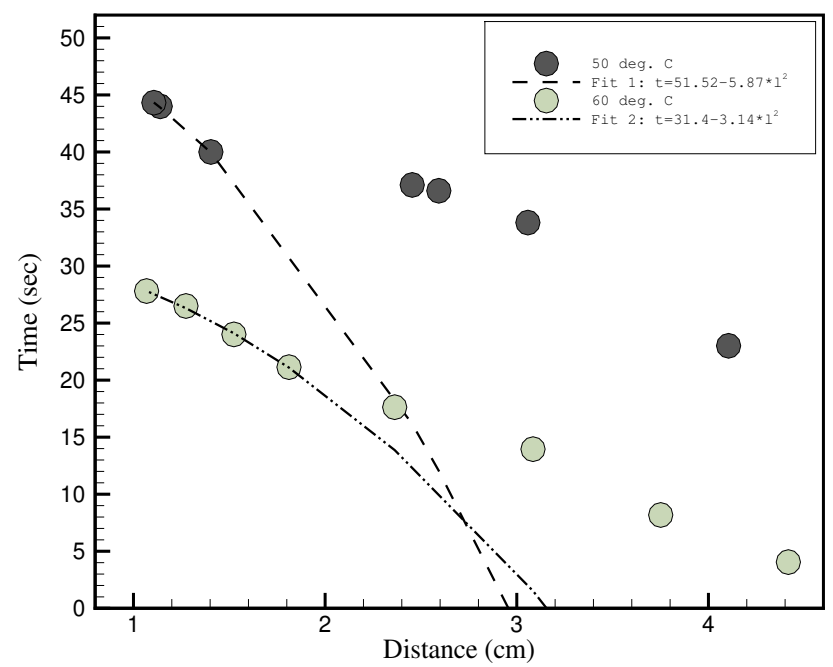

Figure 5: Time as a function of the distance between the center of two spheres for two choices of temperature. The spheres are seen to aggregate faster at higher temperatures. The experimental data is fit to the asymptotic curve given by Eq. 4. Clearly, the curves reveal a better fit in the asymptotic regime where the initial separation between the spheres is small.

\section{Discussion}

Determining an exact expression for $t_{a}$ as a function of $\theta$ is rather challenging. We therefore begin with the simple analytical expression provided in the literature $[4,3,1]$ and consider an appropriate modification based upon our experiments. At the outset aggregation is the result of the competition between the attractive capillary force and retarding viscous forces imposed on the floating bodies. Based on analysis by Chan et al. [4] the force between two spheres of radii $R_{1}$ and $R_{2}$ can be given by

$$
F(l)=-2 \pi \gamma\left(R_{1} R_{2}\right)^{\frac{1}{2}}\left(B_{1} B_{2}\right)^{\frac{5}{4}}\left(\Sigma_{1} \Sigma_{2}\right) K_{1}(\lambda l),
$$

where $\gamma$ is the surface tension, $B_{i}=\frac{g R_{i}^{2} \rho}{\gamma}(i=1,2)$ is the Bond number, $\Sigma_{i}(i=1,2)$ is a dimensionless parameter depending on the contact angle $\theta_{i}(i=1,2)$ given by

$$
\Sigma_{i}=\frac{2}{3} D_{i}-\frac{1}{3}-\frac{1}{2} \cos \theta_{i}+\frac{1}{6} \cos ^{3} \theta_{i}
$$


and $K_{1}(x)$ is a modified Bessel function of the first kind. In the expression for the Bond number, $g$ is the acceleration due to gravity, and $\rho$ is the fluid density. In the expression for $\Sigma_{i}$, the quantity $D_{i}$ is the density ratio and is given by $D_{i}=\frac{\rho_{i}-\rho_{\text {air }}}{\rho_{\text {fluid }}-\rho_{\text {air }}}(i=1,2)$, where $\rho_{i}$ is the density of the $i^{\text {th }}$ sphere $(i=1,2)$. In the Bessel function argument, $l$ is the interaction distance between two spheres and $\lambda$ is the inverse capillary length. In a recent study on the aggregation of spheres floating on a liquid surface, Vella and Mahadevan [1] consider the force balance equation for the aggregation dynamics. For the case of two different spheres, the equation can be written as

$$
6 \pi \mu R_{1} \alpha \frac{d l}{d t}=-2 \pi \gamma\left(R_{1} R_{2}\right)^{\frac{1}{2}}\left(B_{1} B_{2}\right)^{\frac{5}{4}}\left(\Sigma_{1} \Sigma_{2}\right) K_{1}(\lambda l)
$$

where the left hand side represents the Stokes drag term. If we approximate $\lambda l<<1$, then the Bessel function $K_{1}(\lambda l) \approx \frac{1}{\lambda l}$. In this limit of small separation, we can easily integrate the asymptotic form of Eq. 3 to obtain the following expression for time of contact:

$$
t_{a} \approx \frac{3 \mu \alpha \sqrt{R_{1}}\left(l_{0}^{2}-l^{2}\right)}{2 \gamma l_{c}\left(B_{1} B_{2}\right)^{\frac{5}{4}}\left(\Sigma_{1} \Sigma_{2}\right) \sqrt{R_{2}}},
$$

where $\mu$ is the coefficient of viscosity, $\alpha$ is the drag coefficient on the sphere due to its fractional submersion in the liquid, and $\frac{1}{\lambda}=l_{c}=\sqrt{\frac{\gamma}{g \rho}}$ is the capillary length. Equation 4 has the form $y=A-B x^{2}$. This quadratic is used to fit experimental data and the results are shown in Fig. 5. The curves are seen to naturally fit better when the separation distance between the spheres is small.

Based on our experimental study and empirical estimates of the temperature dependence of viscosity and surface tension, we argue that $t_{a}$ should be expressible in terms of the temperature. Based on the literature, all other parameters including the contact angle can be taken to be independent of temperature [25, 26, 27]. Expansion of $B_{i}$ and $l_{c}$ in $\gamma$, followed by a substitution of these expressions into Eq. 4 leads to

$$
t_{a}=c_{0} \mu \gamma
$$

where

$$
c_{0}=\frac{3 \alpha\left(l_{0}^{2}-l^{2}\right)}{2 \rho^{2} g^{2} R_{1}^{2} R_{2}^{3}\left(\Sigma_{1} \Sigma_{2}\right)} .
$$

If one uses $\mu=3.16 \theta^{-0.775}$ and $\gamma(\theta)=93.8 \theta^{-0.15}$ in equation (5), we have $t_{a} \propto \theta^{-0.925}$. In fact, based on the range for $\gamma$ from our surface tension experiments, we have $t \propto \theta^{-s}$ where $0.783 \leq s \leq 1.125$.

One can also use a similar argument to very approximately estimate aggregation time of multiple $(N)$ spheres. In order to do so, we use the expression for capillary force between a sphere and infinite cylinder derived by Chan et al. [4]. The infinite cylinder is considered to approximate the $N-1$ remaining spheres in the system which are assumed to have aggregated first. While this model does not accurately reflect the experimental scenario of $N$ spheres aggregating it makes an interesting comparison with respect to the first model. The capillary force between the sphere and a cylinder, in this instance, is given by the expression

$$
F(l)=-\pi \gamma R_{s} B_{s} B_{c} \Sigma_{s} \Sigma_{c} e^{-\lambda l},
$$


where $R_{s}$ is the radius of the sphere and $B_{s}$ and $B_{c}$ correspond to Bond numbers for the sphere and cylinder respectively. $\Sigma_{s}$ is as defined in equation (2) while $\Sigma_{c}$ is given by the expression

$$
\Sigma_{c}=\pi\left(D_{i}-1\right)+\theta_{i}-\frac{1}{2} \sin 2 \theta_{i}
$$

for $i=1,2$. The force balance equation for the sphere-cylinder system now becomes

$$
6 \pi \mu \alpha \frac{d l}{d t}=-\pi \gamma R_{s} B_{c} B_{s} \Sigma_{s} \Sigma_{c} e^{-\lambda l}
$$

which upon integration yields the exact result

$$
t_{a}=\left(\frac{6 \alpha\left(e^{-\lambda l}-e^{-\lambda l_{0}}\right)}{g^{2} \rho_{s} \rho_{c} R_{s}^{2} R_{c}^{2} \Sigma_{s} \Sigma_{c}}\right) \mu \gamma .
$$

Again, Eq. (9) shows a decaying profile with increasing temperature, $t_{a} \propto \theta^{-s}$ where $0.783 \leq$ $s \leq 1.125$.

\section{Conclusions}

In summary, we have conducted an experimental study to investigate the impact of temperature upon the surface tension driven aggregation of floating spheres. The observations resulting from these simple experiments are in agreement with similar observations about aggregation of floating bodies in the literature, although controlled experiments to determine the systematic effect of temperature have not been conducted. We show that thermal effects are revealed in this phenomenon by changing the viscosity and surface tension of the liquid. The reduced effective viscosity of the liquid, with increasing temperature, has the overall impact of reducing the aggregation time. We realize that our theoretical estimates of $t_{a}$ versus $\theta$ do not reveal the decay profile seen in our experiments; convection driven surface flow, which is neglected here, could be the cause of the observed mismatch. More rigorous theoretical and numerical studies are needed to confirm these missing effects.

\section{Acknowledgment}

EF is supported by the National Science Foundation award DMS \#1418956. LH is currently an undergraduate student at Harvard University; he contributed to this project while a high school student at Montclair High School. AV would also like to thank Dr. Vijay Rangachari for helpful comments.

\section{References}

[1] D. Vella and L. Mahadevan, Am. J. Phys., 73 (9), 2005, 817-825.

[2] M. M. Nicolson, Proc. Cambridge Philos. Soc. 45, 1949, 288295. 
[3] W. A. Gifford, L. E. Scriven, Chem Eng Sci, 26, 1971, 287297.

[4] D. Y. C. Chan, J. D. Henry Jr., and L. R. White, J. Colloid Interface Sci. 79, 1981, 410418.

[5] P. Singh and D. D. Joseph, J. Fluid Mech., 530, 2005, 31-80.

[6] H. N. Dixit and G.M. Homsy, Physics of Fluids, 24, 2012, 122102.

[7] N. Aubry, P. Singh, M. Janjua and S. Nudurupati, Proc. Natl. Acad. Sci., 105 (10), 2008, 37113714.

[8] W. Evans, R. Prasher, J. Fish, P. Meakin, P. Phelan and P. Keblinski, International Journal of Heat and Mass Transfer, 51, Issue 5-6, 2008, 1431-1438.

[9] D. D. Joseph, J. Wang, R. Bai, B.H. Yang and H.H. Hu, Journal of Fluid Mechanics, 496, 2003, 139-163.

[10] L. Liu, W. Qi,D. K. Schwartz, T. W. Randolph and J. F. Carpenter, Journal of Pharmaceutical Sciences, 102 (8), 2013, 24602470.

[11] Surface Activity of Proteins: Chemical and Physicochemical Modifications. Edited by S. Magdassi, Marcel Dekker, Inc., New York, Basel, Hong Kong 1996.

[12] C. Schmitt, C. Bovay ,M. Rouvet, S.Shojaei-Rami and E. Kolodziejczyk, Langmuir, 23 (8), 2007, 41554166.

[13] Born, P., Murray, E. and Kraus, T., J. Phys. Chem. Solids, 71, 95, 2010.

[14] Wink, T., van Zuilen, S.J., Bult, A. and van Bennkom, W.P., Analyst,122,43R,1997.

[15] equsa, S., Redmond, P.L. and Scherer, N.F., J. Phys. Chem. C, 111, 17993, 2007.

[16] Pochan, D.J., Schneider, J.P., Kretsinger, J., Ozbas, B., Rajagopal, K. and Haines, L., J. Am. Chem. Soc., 125, 11802, 2003.

[17] Qin, S., Geng, Y., Discher, D.E. and Yang, S., Adv. mater., 18, 2905, 2006.

[18] Sharma, R., Langmuir, 23, 6843, 2007.

[19] Valkama, S., Kosonen, H., Ruokolainen, J., Haatainen, T., Torkkeli, M., Serimaa, R., Ten Brinke, G. and Ikkala, O., Nat. mater., 3, 872, 2004.

[20] Chabanov, A.A., Jun, Y. and Norris, D.J., Appl. Phys. Lett., 84, 3573, 2004.

[21] C. J. Seeton, Viscositytemperature correlation for liquids, Tribology Letters, 22 (1), 2006 , 67-78.

[22] Cini, R., Loglio, G. and Ficalbi, A., Journal of Colloid and Interface Science, 41, 287-297, 1972. 
[23] Gittens, G., Journal of Colloid and Interface Science, 30, 406, 1969.

[24] Kensington, A., The Physics and Chemistry of Surfaces,3rd edition, Oxford University Press, 1941.

[25] Bernardin, J., Mudawar, I., Walsh, C. and Franses, E., Int. J. heat and Mass Transfer, 40, 1017, 1997.

[26] Jones, J. and Adamson, A., J. Phys. Chem., 72, 646, 1968.

[27] Phillips, M. and Riddiford, A., Nature, 205, 1017, 1965. 